\title{
Alveolar adenoma of the lung: multidisciplinary case discussion and review of the literature
}

\author{
Leonid Roshkovan $^{1 \wedge}$, Jeffrey C. Thompson ${ }^{2}$, Sharyn I. Katz ${ }^{1}$, Charuhas Deshpande ${ }^{3}$, Taylor Jenkins ${ }^{3}$, \\ Anna K. Nowak ${ }^{4}$, Rosyln Francis ${ }^{5}$, Carole Dennie ${ }^{6}$, Dominique Fabre ${ }^{7}$, Sunil Singhal ${ }^{8}$, \\ Maya Galperin-Aizenberg ${ }^{1}$
}

\begin{abstract}
${ }^{1}$ Division of Thoracic Radiology, University of Pennsylvania Perelman School of Medicine, Philadelphia, PA, USA; ${ }^{2}$ Division of Pulmonary, Allergy and Critical Care, University of Pennsylvania Perelman School of Medicine, Philadelphia, PA, USA; ${ }^{3}$ Department of Pathology and Laboratory Medicine, University of Pennsylvania Perelman School of Medicine, Philadelphia, PA, USA; ${ }^{4}$ Medical School, University of Western Australia, Perth, Australia; ${ }^{5}$ Department of Nuclear Medicine, Sir Charles Gairdner Hospital, Perth, Australia; ${ }^{6}$ Department of Medical Imaging, The Ottawa Hospital, University of Ottawa, Ottawa, Canada; ${ }^{7}$ Department of Thoracic Surgery, Marie Lannelongue Hospital, Paris Sud Saclay University, Paris, France; ${ }^{8}$ Department of Surgery, University of Pennsylvania Perelman School of Medicine, Philadelphia, PA, USA

Correspondence to: Leonid Roshkovan, MD. Department of Radiology, Hospital of the University of Pennsylvania, 1 Silverstein Bldg, 3400 Spruce St., Philadelphia, PA 19104, USA. Email: leonid.roshkovan@pennmedicine.upenn.edu.
\end{abstract}

Submitted May 03, 2020. Accepted for publication Sep 07, 2020.

doi: $10.21037 /$ jtd-20-1831

View this article at: http://dx.doi.org/10.21037/jtd-20-1831

\section{Introduction}

Indeterminate pulmonary nodules detected on imaging are a common management challenge with cases often referred for multidisciplinary discussion to determine the best course of action often a decision between surgical intervention or longitudinal follow-up. Beyond malignancy, there are a number of benign pulmonary nodules that are detected on imaging and present a management dilemma when these lesions are large. Though international guidelines such as that published by the Fleischner society (1) and British Thoracic Society (2) generally recommend surveillance for small incidental solid pulmonary nodules, when solid pulmonary nodules are larger than a centimeter in size there is an increased likelihood to intervene surgically due to an increased risk of malignancy and patient anxiety.

Alveolar adenoma is a rare and usually benign pulmonary tumor often discovered incidentally on cross-sectional imaging. Approximately 40 cases have been described in the literature with few reports documenting long-term imaging surveillance on both computed tomography (CT) and ${ }^{18} \mathrm{~F}$-fluorodeoxyglucose $\left({ }^{18} \mathrm{FDG}\right)$-positron emission tomography (PET)/CT both modalities that are frequently employed in the characterization of pulmonary nodules.

We report a case discussion regarding an incidentally detected $12 \mathrm{~mm}$ solid-appearing pulmonary nodule in a 48 -year-old female followed by 2 years of imaging surveillance on thoracic CT and ${ }^{18} \mathrm{FDG}$ PET/CT prior to surgical resection, which revealed that this was a benign alveolar adenoma on final pathology. We also review and summarize the imaging appearance of this tumor across multiple cross-sectional imaging modalities, and we have invited an international multidisciplinary team to discuss management of large pulmonary nodules exhibiting an indolent pattern of growth, a commonly encountered clinical management challenge.

\section{Case presentation}

A 48-year-old female non-smoker with a history of colonic tubulovillous adenoma status post hemicolectomy and a pituitary adenoma, that resolved spontaneously following pregnancy, presented to our institution with a $12-\mathrm{mm}$ lung nodule incidentally discovered on a diagnostic CT of the chest performed for chest pain.

\footnotetext{
$\wedge$ ORCID: 0000-0002-8890-8835.
} 

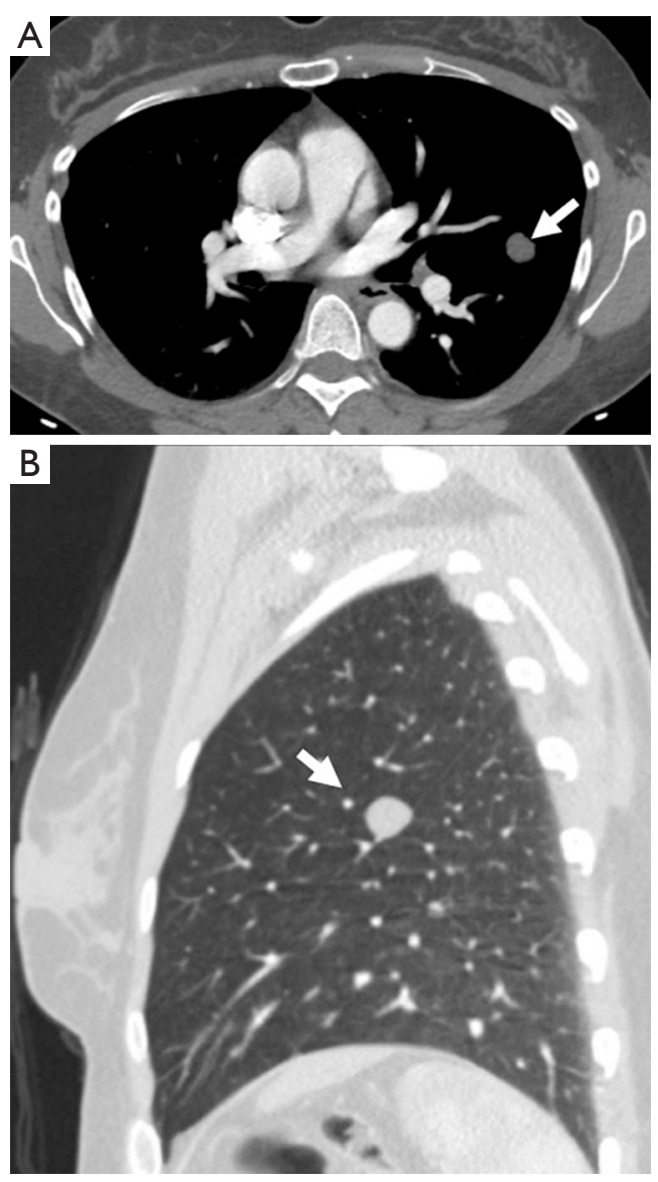

Figure 1 Contrast enhanced CT of the chest in (A) axial plane with soft tissue windows and (B) sagittal plane with lung windows obtained at presentation. Images reveal a well-defined, smoothly marginated nodule.

The initial contrast-enhanced chest CT (Figure 1) acquired in the arterial phase revealed a smoothly marginated solid-appearing juxta-pleural nodule in the left upper lobe measuring $12 \mathrm{~mm}$ in maximal diameter with mean attenuation value in the range of fluid/fat (-3.5 Hounsfield units). A subsequent ${ }^{18}$ FDG PET/ CT (Figure 2) performed 2 days later demonstrated that this lesion had no significant ${ }^{18} \mathrm{FDG}$ avidity, with a standard uptake value (SUV)max of 0.6 which measured only slightly above the lung parenchymal background. A follow-up contrast-enhanced CT scan performed 7 months later obtained in the portovenous phase of injection demonstrated interval stability in size of the nodule with slightly higher attenuation values with a mean
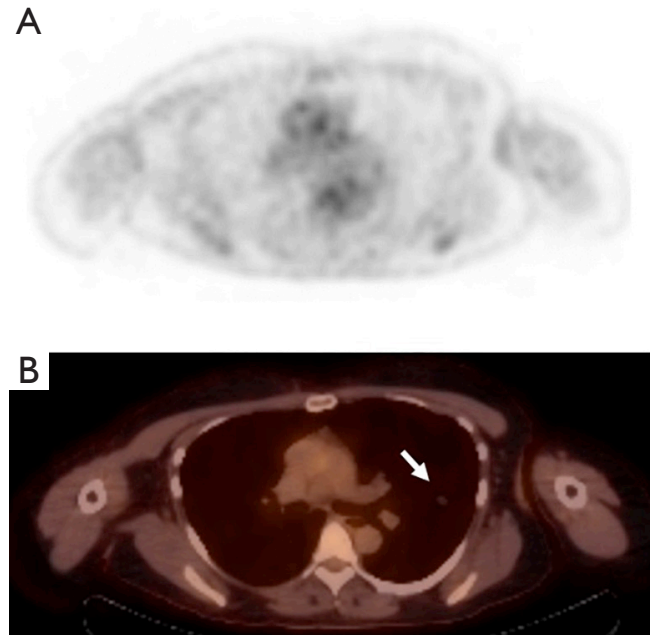

Figure 2 Whole body FDG PET-CT. (A) Axial attenuation corrected 18FDG-PET image and (B) fused 18FDG -PETCT image obtained 2 days after initial CT scan demonstrates no significant uptake of FDG in the nodule marked by the arrow.

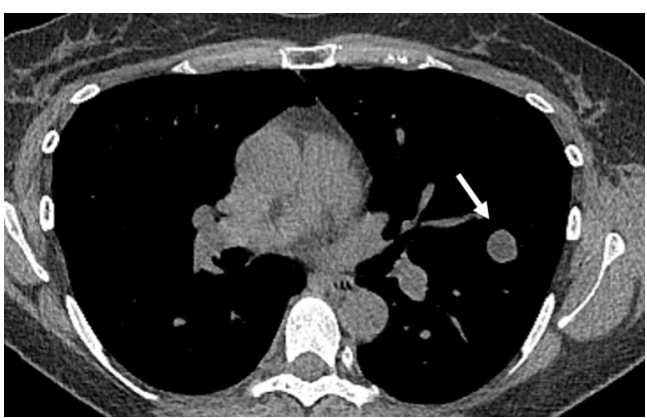

Figure 3 Axial unenhanced CT scan of the chest obtained 2 years after initial CT reveals no appreciable change in the size or texture of the nodule marked by the arrow.

CT attenuation of 7 Hounsfield units, which is in the range of fluid attenuation. This nodule continued to be stable on unenhanced CT chest performed at 23 months from initial presentation (Figure 3) at which time it demonstrated a mean attenuation value of -26 Hounsfield units which is in the range of fluid/fat attenuation.

Given the long-term stability and indolent imaging behavior, continued observation was initially recommended, however due to ongoing patient concern, this case was presented at our institutional multidisciplinary tumor board to discuss management options. 


\section{International Multidisciplinary Team (IMDT) discussion}

Expert 1: (chest radiologist \#1)

This is an interesting multi-modality case presentation of a very rare benign tumor of the lung. The imaging characteristics and 2-year follow-up suggest a benign lesion. The differential diagnosis of a smoothly-marginated lung nodule with fluid/fat attenuation most commonly includes an intrapulmonary bronchogenic cyst and hamartoma. Alveolar adenoma would be a rare addition to this differential. Although sub-solid nodules which can represent lung adenocarcinomas should be followed for a longer period than 2 years due to their indolent growth pattern, the lack of growth in a solid or low-density smooth, round nodule for 2 years can safely be assumed to be benign. Even carcinoid tumors tend to demonstrate visible growth over 2 years and do not demonstrate fluid or fat attenuation on CT. Carcinoid tumors may not have increased FDG uptake on PET/CT similar to benign lesions but typically demonstrate enhancement on CT due to their vascular nature. Enhancement was not seen in the current case presented.

\section{Expert 2 (Surgeon \#1)}

The fact that this nodule had been present for 2 years with minimal changes in a young female who was a non-smoker suggested it was benign in nature. Differential diagnostic consideration for this situation includes a carcinoid, hamartoma, atypical adenomatous hyperplasia, sclerosing hemangioma, and a mucoepidermoid carcinoma. An alveolar adenoma is a rare disease and would not be the initial suspected pathology. Another concern if a biopsy had been successful is the possibility of malignant transformation if the adenoma was suspicious for a mucinous adenocarcinoma or a minimally invasive adenocarcinoma.

In considering methods for potential biopsy, computed tomography (CT)-guided pulmonary core biopsies of small pulmonary nodules less than $10 \mathrm{~mm}$ are challenging for interventional radiologist, and their diagnostic accuracy has been shown to be variable in previous studies. But it is a safe technique. The risk of pneumothorax and alveolar hemorrhage is more important for lesion in the lower lobe. Robotic Assisted Bronchoscopy (RAB) is another diagnostic option for small nodules. This technique includes electromagnetic navigation, radial probe ultrasonography, ultrathin bronchoscopy or virtual bronchoscopy remains suboptimal. RAB may be beneficial for patients with peripheral lung lesions that require biopsy prior to surgical resection or to avoid surgery.

\section{Expert 3 (Surgeon \#2)}

Given the slow growing nature and lack of FDG avidity suggested a lobectomy would not be indicated unless the frozen section was confirmatory for a lung carcinoma. During the operation, a complete wedge of the nodule was performed with grossly negative margins. Although the patient's pulmonary function tests were well within the acceptable range, there was some value in preserving as much lung function as possible until the final diagnosis was reached on permanent section.

\section{Expert 4 (Medical oncologist \#1)}

This case describes identification and follow up of a solitary pulmonary nodule $(\mathrm{SPN})>8 \mathrm{~mm}$ in diameter in a patient with no apparent risk factors for pulmonary malignancy. In order to further risk-stratify, information on passive smoking, family lung cancer history, smokeless electronic cigarette use, and asbestos exposure would be useful to supplement knowledge of lesion morphology, location and size. The smoothly marginated solid appearance and juxtapleural location reduce the likelihood of malignancy, however the $12 \mathrm{~mm}$ size mandated follow up. Additional investigation with any of 3-month CT, FDG-PET-CT, or lesion biopsy would have been appropriate. The lack of ${ }^{18}$ FDG avidity coupled with subsequent stability over 23 months is in keeping with a benign lesion, and guidelines would support no requirement for further imaging follow up. However, in this case the patient may have found no further follow up distressing; further regular imaging follow up would have led to radiation exposure. Seeking a histological diagnosis was a pragmatic, individualized decision.

\section{Expert 5 (Nuclear Medicine Radiologist \#1)}

${ }^{18}$ FDG PET/CT imaging for the evaluation of a solitary pulmonary nodule is performed to ascertain the FDGavidity of the lesion, as highly FDG-avid lesions are associated with an increased risk of malignancy. In this case, low FDG-avidity is reassuring, especially in the context of low clinical probability of malignancy. The sensitivity of PET for detection of malignant lung lesions is influenced 
by lesion size, however a rounded lesion of $12 \mathrm{~mm}$ is of sufficient size for evaluation with ${ }^{18} \mathrm{FDG}$ PET/CT, and a SUVmax of 0.6 would favor a non-aggressive lesion. By performing a whole body ${ }^{18} \mathrm{FDG}$ PET/CT scan, additional unsuspected lesions have been excluded, including the possibility of an occult primary malignancy with a metastatic lung lesion.

\section{Expert 6 (Pulmonologist \#1)}

The case describes a 48-year-old female presenting for evaluation of an incidentally detected $12 \mathrm{~mm}$ solid nodule. The management of a solitary pulmonary nodule should be guided by first assessing the probability of malignancy for a given nodule based on the nodule characteristics and patient-specific risk factors. Specific nodule features that should be taken into consideration when estimating the probability of malignancy include the nodule size, morphology, growth rate, and location. Certain patient characteristics including age, smoking status, prior cancer history, occupational exposures (e.g., asbestos), family history of lung cancer, and evidence emphysema or pulmonary fibrosis are associated with an increased risk of malignancy and should also be evaluated. In this case, the patient is a young, non-smoker with a nodule that is smoothly-marginated, lacks FDG avidity, and is unchanged in size over a 23 -month period, all of which suggests a benign etiology. However, carcinoid tumors may have this morphologic appearance and can be slow growing over time. Although one would expect a carcinoid tumor to have some growth over this period of time as well as some enhancement on a contrasted study, would recommend additional surveillance imaging in 12 months given an indolent carcinoid tumor remains in the differential diagnosis.

\section{Treatment and follow-up}

Due to the patient's persistent concern, the decision was made to move forward with surgical resection. She underwent a video-assisted thoracoscopic wedge resection of the left upper lobe without complications. Pathology of the resected specimen (Figure 4) demonstrated a wellcircumscribed lesion composed of cystically dilated spaces filled with eosinophilic fluid, separated by septae with myxoid stroma and lined by low cuboidal cells (Figure 4). Immunohistochemical stains revealed these low cuboidal cells to be positive for thyroid transcription factor 1 (TTF-1).
Special stains for periodic acid-schiff (PAS) and PAS diastase stain highlighted PAS-positive material within these cystically dilated spaces. The overall morphology and staining results supported the diagnosis of an alveolar adenoma.

\section{Discussion}

Management of incidentally detected pulmonary nodules is a commonly encountered clinical problem which can be challenging when nodules are large at presentation. Imaging features that suggest a benign histology include smooth borders, indolent pattern of growth, poor ${ }^{18} \mathrm{FDG}$ avidity and the presence of fat or dense calcification. We have presented a case of a $12-\mathrm{mm}$ nodule with indolent imaging features which ultimately was resected revealing alveolar adenoma, an extremely rare pulmonary neoplasm, first described in 1986 by Yousem et al. (3). Usually this entity presents as a single lesion incidentally detected on plain radiograph or cross-sectional imaging. There are a few case reports that describe multiple lesions detected on imaging, although pathologic confirmation was obtained for only a single lesion in each case with the presumption that the additional lesions represented the same pathological entity (4). This lesion is predominantly found in middleaged women and typically demonstrates a peripheral and subpleural location, however more central locations have occasionally been described $(5,6)$. Although alveolar adenomas are generally regarded as benign, there is a single case report describing the malignant transformation of an alveolar adenoma into an adenocarcinoma in an elderly patient (7).

On histopathological examination, these tumors usually demonstrate multiple cystic spaces lined with type 2 pneumocytes that stain positively for thyroid transcription factor-1 (TTF-1) with intermixed interstitial stroma that contain mostly fibroblasts that stain positively for vimentin and CD34 $(8,9)$. Characteristic imaging features include a well-circumscribed lesion with smooth borders and homogeneous low attenuation; although several case reports describe nodules with small cystic spaces, and a single case report describes a symptomatic cavitary mass $(4,10)$.

Alveolar adenomas have been described to have several enhancement patterns after intravenous contrast injection on CT with most cases demonstrating no enhancement and a minority of cases demonstrating spotty or mild enhancement, similar to what was observed in this patient (11). With regards to ${ }^{18} \mathrm{FDG}$ activity, a total of 6 case 


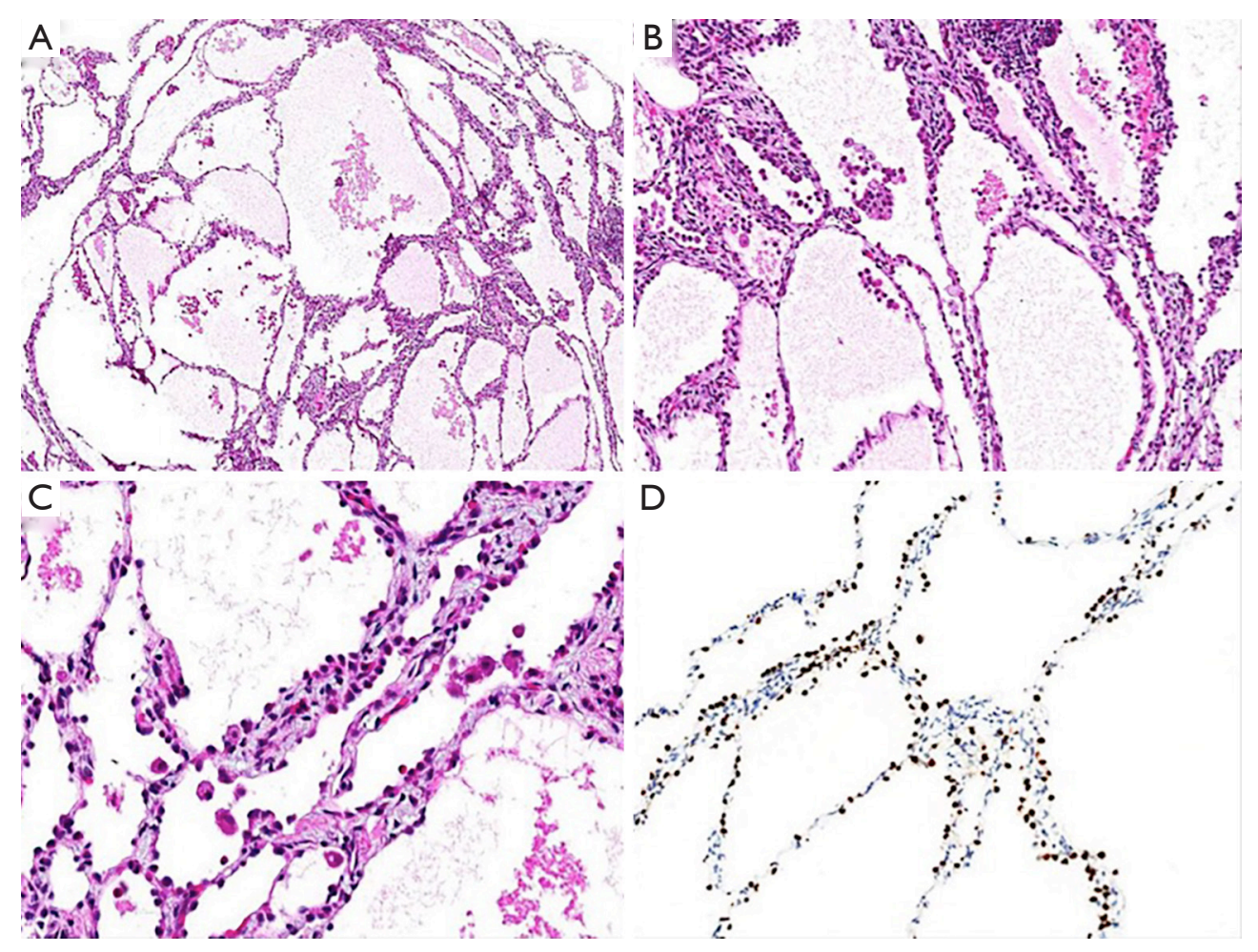

Figure 4 Hematoxylin and Eosin stain. Pathology specimen reveals a multi-cystic lesion composed of cysts of various sizes with a proliferation of type II pneumocytes lining alveolar-like septae (A, 50x; B, 100x). The alveolar-like spaces are filled with a granular, eosinophilic material (B, 100x). Higher power demonstrates the cyst-lining hobnailed, cuboidal pneumocytes and the bland spindle cells with a myxoid matrix within the septae (C, 200x). The type II pneumocytes lining the cysts show diffuse TTF-1 nuclear positivity (D, 100×).

reports, including ours, used ${ }^{18}$ FDG-PET or ${ }^{18}$ FDG -PET/ $\mathrm{CT}$ in the imaging evaluation of this lesion. Three cases did not demonstrate significant FDG activity similar to our case, and two cases demonstrated minor ${ }^{18} \mathrm{FDG}$ avidity $(4,5,7,10)$. These uptake features overall favor an indolent biologic behavior, although it is important to keep in mind that some malignant tumors such as well differentiated adenocarcinomas and carcinoids are known to have lower than expected or no ${ }^{18}$ FDG uptake $(12,13)$.

Magnetic resonance imaging (MRI) was used for the characterization an alveolar adenoma lesion in four case reports. One described imaging characteristics similar to other cystic lesions in the body with low signal intensity on $\mathrm{T} 1$, high signal intensity on $\mathrm{T} 2$ and rim enhancement after intravenous contrast injection (14). Two others describe the presence of central low signal intensity on T1-weighted sequences with intermediate signal in the periphery of the lesion and a high signal intensity centrally with low signal intensity in the periphery on T2-weighted sequences that did not demonstrate enhancement after contrast injection (15).

\section{Expert 1 (Radiologist \#1): would you have considered biopsy of this lesion to obtain a diagnosis rather than surgical intervention?}

In our institution and in others where the expertise is available, image-guided needle biopsy is an excellent technique for diagnosis of benign lung nodules when patients are uncomfortable with the prospect of longterm imaging observation. Core needle biopsy has higher diagnostic accuracy than fine needle aspiration for the specific diagnosis of benign lesions and is thus the preferred technique for tissue diagnosis when benign lesions are suspected (16). Even in the absence of a specific benign diagnosis, a biopsy that is "negative for malignancy" can be very reassuring for patients especially in the absence of lesion growth on follow-up imaging. The risk from an image-guided biopsy is also much lower than that of surgery and would have been the recommended management 
option for this patient at our institution.

\section{Expert 2 (Surgeon \#1): why did you choose to resect the nodule versus continued surveillance?}

Given the limited information on these nodules, we have suggested to the patient to obtain a CT scan every 2 to 3 years for surveillance. We do not believe more frequent imaging is indicated for surveillance. During surveillance evaluating not only nodule diameter but also volume is also of interest. Even small changes in the maximum diameter can result in significant changes in volume. Absolute nodule volume and increases in nodule volume has been associated with an increased likelihood of malignancy (17).

\section{Expert 3 (Surgeon \#2): what is your long-term follow up plan?}

The patient's anxiety was a significant factor in this decision. The patient had young children that she was responsible for, thus, she did not want to receive repeat surveillance CT scans, despite the fact that the patient was re-assured. In this case the decision was made to resect however we would like to emphasize that it is vital that this shared decisionmaking be made as an evidence based discussion. In this case, it would have been entirely acceptable to follow this lesion non-invasively with imaging rather than resection.

\section{Expert 4 (Radiologist \#2): will you recommend standard dose, low dose or ultra-low-dose CT scan be used for her imaging surveillance?}

Given that this nodule is $12 \mathrm{~mm}$ in size, and no other nodules are present that require surveillance in this nonsmoker, a low dose CT could be obtained for surveillance. In this case, the nodule has been resected and found to be benign, no further imaging surveillance would be recommended.

\section{Conclusions}

Incidental pulmonary nodules are a commonly encountered diagnostic and management dilemma, particularly when large in size. In the absence of risk factors for malignancy, small nodules are often followed with CT surveillance $(1,2)$. However larger nodules can be more challenging to manage due to a theoretical risk of malignancy with patients often undergoing surgical resection. We have presented a case of an indolent $12 \mathrm{~mm}$ nodule that was ultimately resected and found to be an alveolar adenoma. This is an extremely rare and usually benign pulmonary neoplasm that is often incidentally discovered on cross-sectional imaging. Imaging characteristics indicating an indolent biology are typically present including smooth borders, minimal to no enhancement after intravenous contrast injection and absent or minimal ${ }^{18}$ FDG avidity. In our case discussion, we describe the long-term stability in size and appearance of an alveolar adenoma and suggest that in a low-risk patient population with suggestive imaging characteristics, this benign neoplasm should be considered in the differential diagnosis of indolent lesions and that surveillance with serial imaging can be considered in the management of these patients. Finally, the options expressed in this manuscript are those of the authors and we recognize that practices may vary by practice setting.

\section{Acknowledgments}

Funding: None.

\section{Footnote}

Conflicts of Interest: All authors have completed the ICMJE uniform disclosure form (available at http://dx.doi. org/10.21037/jtd-20-1831). AKN reports grants, research funding to institution and travel funding 2019 from Astra Zeneca; grants, research funding to institution, clinical trials consulting and payment to institution from Douglas Pharmaceuticals; other from Bayer Pharmaceuticals, other from Roche Pharmaceuticals, other from Boehringer Ingelheim, other from Merck Sharpe Dohme, other from Pharmabcine, other from Atara Biotherapeutics, other from Trizell Ltd, outside the submitted work. The other authors have no conflicts of interest to declare.

Ethical Statement: The authors are accountable for all aspects of the work in ensuring that questions related to the accuracy or integrity of any part of the work are appropriately investigated and resolved. The subject of this case report and multidisciplinary discussion gave informed consent before taking part in this manuscript.

Open Access Statement: This is an Open Access article distributed in accordance with the Creative Commons Attribution-NonCommercial-NoDerivs 4.0 International License (CC BY-NC-ND 4.0), which permits the non- 
commercial replication and distribution of the article with the strict proviso that no changes or edits are made and the original work is properly cited (including links to both the formal publication through the relevant DOI and the license). See: https://creativecommons.org/licenses/by-nc-nd/4.0/.

\section{References}

1. MacMahon H, Naidich DP, Goo JM, et al. Guidelines for Management of Incidental Pulmonary Nodules Detected on CT Images: From the Fleischner Society 2017. Radiology 2017;284:228-43.

2. Baldwin DR, Callister ME, Guideline Development G. The British Thoracic Society guidelines on the investigation and management of pulmonary nodules. Thorax 2015;70:794-8.

3. Yousem SA, Hochholzer L. Alveolar adenoma. Hum Pathol 1986;17:1066-71.

4. Kondo N, Torii I, Hashimoto M, et al. Alveolar adenoma of the lung: a case report. Ann Thorac Cardiovasc Surg 2011;17:71-3.

5. Nosotti M, Mendogni P, Rosso L, et al. Alveolar adenoma of the lung: unusual diagnosis of a lesion positive on PET scan. A case report. J Cardiothorac Surg 2012;7:1.

6. Wang L, Wang X, Rustam A, et al. Alveolar adenoma resected by thoracoscopic surgery. Ann Thorac Cardiovasc Surg 2013;19:489-91.

7. Okada S, Ohbayashi C, Nishimura M, et al. Malignant transformation of alveolar adenoma to papillary adenocarcinoma: a case report. J Thorac Dis 2016;8:E358-61.

8. Burke LM, Rush WI, Khoor A, et al. Alveolar adenoma: a histochemical, immunohistochemical, and ultrastructural

Cite this article as: Roshkovan L, Thompson JC, Katz SI, Deshpande C, Jenkins T, Nowak AK, Francis R, Dennie C, Fabre D, Singhal S, Galperin-Aizenberg M. Alveolar adenoma of the lung: multidisciplinary case discussion and review of the literature. J Thorac Dis 2020;12(11):6847-6853. doi: 10.21037/jtd20-1831 analysis of 17 cases. Hum Pathol 1999;30:158-67.

9. De Rosa N, Maiorino A, De Rosa I, et al. CD34

Expression in the Stromal Cells of Alveolar Adenoma. Case Rep Med 2012;2012:913517.

10. Halldorsson A, Dissanaike S, Kaye KS. Alveolar adenoma of the lung: a clinicopathological description of a case of this very unusual tumour. J Clin Pathol 2005;58:1211-4.

11. Petrella F, Rizzo S, Pelosi G, et al. Giant alveolar adenoma causing severe dyspnoea. J Thorac Oncol 2010;5:1088-90.

12. Iwano $S$, Ito $S$, Tsuchiya $K$, et al. What causes falsenegative PET findings for solid-type lung cancer? Lung Cancer 2013;79:132-6.

13. Tatci E, Ozmen O, Gokcek A, et al. 18F-FDG PET/CT rarely provides additional information other than primary tumor detection in patients with pulmonary carcinoid tumors. Ann Thorac Med 2014;9:227-31.

14. Wang X, Li WQ, Yan HZ, et al. Alveolar adenoma combined with multifocal cysts: case report and literature review. J Int Med Res 2013;41:895-906.

15. Fujimoto K, Muller NL, Sadohara J, et al. Alveolar adenoma of the lung: computed tomography and magnetic resonance imaging findings. J Thorac Imaging 2002;17:163-6.

16. Gong Y, Sneige N, Guo M, et al. Transthoracic fine-needle aspiration vs concurrent core needle biopsy in diagnosis of intrathoracic lesions: a retrospective comparison of diagnostic accuracy. Am J Clin Pathol 2006;125:438-44.

17. Walter JE, Heuvelmans MA, de Jong PA, et al. Occurrence and lung cancer probability of new solid nodules at incidence screening with low-dose CT: analysis of data from the randomised, controlled NELSON trial. Lancet Oncol 2016;17:907-16. 